Article

\title{
In Vivo Ergogenic Properties of the Bifidobacterium longum OLP-01 Isolated from a Weightlifting Gold Medalist
}

\author{
Mon-Chien Lee ${ }^{1,+}$, Yi-Ju Hsu ${ }^{1,+}$, Hsiao-Li Chuang ${ }^{2}{ }^{(D}$, Pei-Shan Hsieh ${ }^{3}$, Hsieh-Hsun Ho ${ }^{3}$, \\ Wei-Ling Chen ${ }^{4}$, Yen-Shuo Chiu ${ }^{5, *}$ and Chi-Chang Huang ${ }^{1, *}$ \\ 1 Graduate Institute of Sports Science, National Taiwan Sport University, Taoyuan City 33301, Taiwan \\ 2 National Laboratory Animal Center, National Applied Research Laboratories, Taipei 11529, Taiwan \\ Glac Biotech Co., Ltd., Tainan City 74442, Taiwan \\ 4 Department of Sports Training Science-Athletics, National Taiwan Sport University, \\ Taoyuan City 33301, Taiwan \\ 5 Department of Orthopedic Surgery, Taipei Medical University Shuang Ho Hospital, \\ New Taipei City 23561, Taiwan \\ * Correspondence: 1021301@ntsu.edu.tw (Y.-S.C.); john5523@ntsu.edu.tw (C.-C.H.); \\ Tel.: +886-3-328-3201 (ext. 2619) (Y.-S.C. \& C.-C.H.) \\ + These authors contributed equally to this work.
}

Received: 27 July 2019; Accepted: 21 August 2019; Published: 24 August 2019

\begin{abstract}
In recent years, probiotics of human origin have shown superior results and performance compared to probiotics from plant or dairy sources, in both in vitro and animal studies. Towards this end, the current study was conducted to explore the ergogenic properties of Bifidobacterium longum subsp. longum OLP-01 isolated from the intestinal microbiome of the gold medalist from the 2008 Beijing Olympics women's $48 \mathrm{~kg}$ weightlifting competition. Male Institute of Cancer Research (ICR) mice were divided into four groups ( $n=10$ per group) and orally administered OLP-01 for 4 weeks at 0 (vehicle), $2.05 \times 10^{9}$ (OLP-01-1X), $4.10 \times 10^{9}$ (OLP-01-2X), and $1.03 \times 10^{10}$ (OLP-01-5X) CFU/kg/day. Physical performance tests including grip strength and endurance time were measured, with OLP-01 supplementation dose-dependently elevating grip strength and endurance. The anti-fatigue activity levels of serum lactate, ammonia, glucose, blood urea nitrogen (BUN), and creatine kinase (CK) were measured after an acute exercise challenge, and OLP-01 was found to significantly decrease lactate, ammonia, and CK levels. OLP-01 treatment was also found to significantly increase the resting levels of both hepatic and muscular glycogen, an indicator of energy storage. Supplementation by OLP-01 showed no subchronic toxic effects while supporting many health-promoting, performance-improving, and fatigue-ameliorating functions.
\end{abstract}

Keywords: olympics; OLP-01; gold medal; probiotic; exercise performance; weightlifting

\section{Introduction}

Exercise increases the alpha diversity of the microbiome of individuals and decreases the thick-walled bacteria, and Clostridium is considered to be one of the main factors regulating the composition and health of intestinal microbial diversity [1]. Exercise promotes the energy harvesting functions of gut microbiota such as antibiotic biosynthesis, amino acid utilization, carbohydrate metabolism, and production of fecal metabolites, e.g., short-chain fatty acids (SCFAs). This leads to enhanced muscle turnover, adaptation, and improved exercise performance. [2]. Athletes have been reported to have a higher diversity of gut microbes, representing 22 distinct phyla, compared to non-athletes with $\mathrm{BMI}<25$ or BMI $>28$ [3]. Exercise can alter the gut microbiota, but there have 
been limited studies showing that athletic performance may be improved by increasing the beneficial microbe in the gut.

The modern definition of probiotic was put forward as an individual or mixed culture of bacteria which, when applied to an animal or human, affects the host beneficially by shaping the indigenous microbiota [4]. There have been many previous studies showing that supplementation of probiotics is able to exert anti-inflammatory properties [5], reduce the incidence of obesity and its related metabolic diseases [6], improve Type 2 diabetes mellitus [7], modulate hypocholesterolemic properties [8], and promote antioxidant responses [9]. There are specific probiotic strains with demonstrated health benefits from the following genera: Lactobacillus, Bifidobacterium, Streptococcus, Pediococcus, Saccharomyces, Bacillus, Leuconostoc, Enterococcus, and Escherichia coli [10]. Most probiotics come mainly from fermented food such as yogurt, fermented milk, cheese, kimchi, granola bar, microalgae, and miso [11]. In recent years, the development and application of human-origin probiotics has increasingly received attention as many studies have shown their superior results and performance in both in vitro and animal studies, compared to probiotics from plant and dairy sources. This appears to be the current trend in the development of probiotics [12,13].

Bifidobacterium longum is a rod-shaped, Gram-positive and catalase-negative bacterium, commonly inhabiting the intestinal tract of humans [14]. B. longum, along with other Bifidobacterium species, colonizes the human gastrointestinal (GI) tract, where it represents up to $90 \%$ of the bacteria of an infant's GI tract [15]. After weaning, this number gradually reduces to $3 \%$ in an adult's GI, and Bacteroides and Eubacterium begin to dominate with age [16]. There have been few previous studies looking at the supplementation of B. longum. Most of these studies have looked at the effect of B. longum mixed with other strains, and have shown improvement in cognitive function [17] and muscular performance [18], reduced obesity [19], and amelioration of colitis and liver injuries [20]. Studies looking at its application and benefit in sport sciences, however, have been limited. Recently, a specific probiotic strain termed B. longum (OLP-01) was isolated from the 2008 Olympic women's $48 \mathrm{~kg}$ weightlifting gold medalist's gut microbiota. We hypothesized that OLP-01 contributes to host energy utilization and mediates the host's characteristic properties in terms of exercise performance, physical fatigue, and body composition. We tested different doses of OLP-01 supplementation on physical performance in vivo, and we then evaluated anti-fatigue function in OLP-01-treated mice.

\section{Materials and Methods}

\subsection{OLP-01 Preparation}

Bifidobacterium longum subsp. Longum OLP-01 was isolated from Wei-Ling Chen, gold medal winner of the 2008 Summer Olympics women's $48 \mathrm{~kg}$ weightlifting competition. The bacterial strain was confirmed by an independent third party, the Food Industry Research and Development Institute (Hsinchu, Taiwan). The dry product of OLP-01 was prepared and provided by Glac Biotech Co., Ltd. (Tainan, Taiwan), and the viable cell count of OLP-01 was $1.07 \times 10^{11} \mathrm{CFU} / \mathrm{g}$. Before supplementation, cells were suspended in phosphate buffered saline (PBS), pH 7.2.

\subsection{Animals and Experimental Design}

Male 7 week old ICR mice under specific pathogen-free conditions were purchased from BioLASCO (Yi-Lan, Taiwan). All animals were provided with distilled water and standard chow diet (No. 5001; PMI Nutrition International, Brentwood, MO, USA) ad libitum, and maintained at a regular cycle (12 h light/dark) at room temperature $\left(23 \pm 2{ }^{\circ} \mathrm{C}\right)$ and $50-60 \%$ humidity. All animal experiments in this study conformed to the guidelines of protocol IACUC-10717 and were approved by the Institutional Animal Care and Use Committee (IACUC) of National Taiwan Sport University.

The human dose of OLP-01, $1 \times 10^{10} \mathrm{CFU}$ per day was modified from previous studies [21]. The doses administered to the mice were converted from a human-equivalent dose (HED) based on body surface area, provided by the U.S. Food and Drug Administration and using the conversion coefficient of 12.3 for the 
mouse species. After a 1 week acclimation period, the 8 week old ICR mice were divided into four groups ( $n=10$ per group) and administered by oral gavage once daily for 4 weeks: (1) vehicle group (0 CFU/kg); (2) OLP-01-1X group (2.05 × 109 CFU/kg); (3) OLP-01-2X group $\left(4.10 \times 10^{9} \mathrm{CFU} / \mathrm{kg}\right)$, and (4) OLP-01-5X group $\left(1.03 \times 10^{10} \mathrm{CFU} / \mathrm{kg}\right)$. All groups were administered the same volume of PBS or supplement and the OLP-01 dose determined according to the body weight of each mouse.

\subsection{Forelimb Grip Strength}

A low-force testing system (Model-RX-5, Aikoh Engineering, Nagoya, Japan) was used to measure the grip strength of mice undergoing vehicle or OLP-01 supplementation. The details have been described previously [22].

\subsection{Exercise Endurance Test}

The swim-to-exhaustion exercise test involved mice carrying constant loads corresponding to 5\% body weight (BW) to analyze endurance time, as previously described [23]. The swimming endurance time of each mouse was recorded from beginning to exhaustion, which was determined by observing loss of coordinated movements and failure to return to the surface within $7 \mathrm{~s}$ [24].

\subsection{Determination of Fatigue-Associated Biochemical Variables}

The fatigue-related biochemical indicators selected to accurately demonstrate physiological status and assess the effects of OLP-01 supplementation were based on our previous reports [25]. The fatigue-associated variables were measured under fasting conditions to reflect the real physiological adaptation under exercise interventions. Blood samples were collected after $10 \mathrm{~min}$ of the swimming exercise and following $20 \mathrm{~min}$ of rest. Samples were centrifuged at $1500 \times \mathrm{g}$ for $10 \mathrm{~min}$ at $4{ }^{\circ} \mathrm{C}$ and serum collected. Serum lactate, ammonia, and glucose levels were determined by an autoanalyzer (Hitachi 7060, Hitachi, Tokyo, Japan). The other variables, such as blood urine nitrogen (BUN) and creatine kinase $(\mathrm{CK})$, were assessed immediately after 90 min extended exercise and 60 min of rest.

\subsection{Resting Biochemical Profiles at the End of the Study}

At the end of the study, all mice were euthanized by $95 \% \mathrm{CO}_{2}$ asphyxiation one hour after the last treatment and blood obtained by cardiac puncture. Serum was collected after centrifugation and biochemical indexes assessed by Hitachi 7060 autoanalyzer. Levels of aspartate aminotransferase (AST), alanine transaminase (ALT), total cholesterol (TC), triglycerides (TG), creatine kinase (CK), glucose (GLU), albumin (ALB), blood urea nitrogen (BUN), creatinine (CREA), uric acid (UA), and total protein (TP) were measured.

\subsection{Body Composition, Glycogen Content, and Histopathology}

After the mice were euthanized, the liver, kidney, heart, lung, muscle, epididymal fat pad (EFP), and brown adipose tissue (BAT) were accurately excised and weighed. Organs were carefully removed, minced, and fixed in 10\% formalin. Tissues were embedded in paraffin and cut into $4 \mu \mathrm{m}$ thick slices for morphological and pathological evaluations. Sections were stained with hematoxylin and eosin (H \& E) and examined under a light microscope equipped with a charge-coupled device (CCD) camera (BX-51, Olympus, Tokyo, Japan) by a clinical pathologist, as previously described [23]. Parts of the muscle and liver tissues were stored in liquid nitrogen for glycogen content analysis, as previously described [26].

\subsection{Bacterial DNA Extraction and $16 S$ rRNA Sequencing}

According to the method previously used by our laboratory, the collected samples were immediately stored at $-80{ }^{\circ} \mathrm{C}$ for DNA extraction after the mice were euthanized. A detailed procedure for sample extraction, preparation, and analysis has been described previously [22]. 


\subsection{Statistical Analysis}

All the statistical analysis was performed using SAS 9.4 (SAS Inst., Cary, NC, USA) and analyzed by one-way analysis of variance (ANOVA). The Cochran-Armitage test was used for the dose-effect trend analysis. Data have been expressed as mean \pm SD with statistical significance set at $p<0.05$.

\section{Results}

\subsection{Effect of OLP-01 Supplementation on Body Weight, Food and Water Intake, and Organ Weights}

The body weight, food intake, water intake, and body compositions are shown in Table 1. The results showed no significant differences in the 4 week growth curves of the vehicle, OLP-01-1X, OLP-01-2X, and OLP-01-5X groups (Figure 1). There was no significant difference in the body weight, food intake, water intake, or body composition among the groups. Only relative EFP weight was significantly lowered by $23.44 \%(p=0.0376), 25.04 \%(p=0.0255)$, and $23.60 \%(p=0.0338)$ with $1 X, 2 X$, and 5X OLP-01 supplementation, respectively, compared to the vehicle group.

Table 1. General characteristics of the experimental groups.

\begin{tabular}{cccccc}
\hline Characteristic & Vehicle & OLP-01-1X & OLP-01-2X & OLP-01-5X & Trend Analysis \\
\hline Initial BW (g) & $30.6 \pm 0.7$ & $30.3 \pm 0.6$ & $30.5 \pm 1.6$ & $30.9 \pm 2.1$ & 0.4253 \\
Final BW (g) & $38.5 \pm 2.3$ & $38.1 \pm 2.7$ & $38.8 \pm 2.9$ & $38.8 \pm 3.2$ & 0.5491 \\
Water intake (mL/mouse/day) & $7.4 \pm 0.7$ & $7.5 \pm 0.8$ & $7.4 \pm 1.0$ & $7.4 \pm 1.4$ & 0.3575 \\
Diet intake (g/mouse/day) & $7.9 \pm 1.1$ & $7.9 \pm 1.0$ & $7.8 \pm 1.8$ & $7.9 \pm 1.5$ & 0.7006 \\
Liver (g) & $2.16 \pm 0.15$ & $2.19 \pm 0.27$ & $2.22 \pm 0.16$ & $2.22 \pm 0.25$ & 0.5576 \\
Muscle (g) & $0.39 \pm 0.03$ & $0.39 \pm 0.03$ & $0.39 \pm 0.03$ & $0.39 \pm 0.05$ & 0.8603 \\
Quadriceps (g) & $0.51 \pm 0.05$ & $0.51 \pm 0.04$ & $0.52 \pm 0.04$ & $0.51 \pm 0.05$ & 0.8678 \\
Kidney (g) & $0.70 \pm 0.09$ & $0.70 \pm 0.08$ & $0.71 \pm 0.12$ & $0.71 \pm 0.09$ & 0.3795 \\
Heart (g) & $0.20 \pm 0.03$ & $0.20 \pm 0.03$ & $0.20 \pm 0.02$ & $0.20 \pm 0.02$ & 0.7699 \\
Lung (g) & $0.23 \pm 0.03$ & $0.24 \pm 0.03$ & $0.23 \pm 0.03$ & $0.24 \pm 0.03$ & 0.8482 \\
EFP (g) & $0.38 \pm 0.16$ & $0.29 \pm 0.10$ & $0.29 \pm 0.09$ & $0.29 \pm 0.07$ & 0.3066 \\
BAT (g) & $0.08 \pm 0.01$ & $0.08 \pm 0.01$ & $0.09 \pm 0.03$ & $0.09 \pm 0.01$ & 0.2921 \\
Cecum (g) & $0.79 \pm 0.20$ & $0.77 \pm 0.05$ & $0.78 \pm 0.10$ & $0.79 \pm 0.16$ & 0.5076 \\
Relative liver weight (\%) & $5.62 \pm 0.15$ & $5.73 \pm 0.32$ & $5.72 \pm 0.12$ & $5.71 \pm 0.23$ & 0.2903 \\
Relative muscle weight (\%) & $1.01 \pm 0.04$ & $1.01 \pm 0.03$ & $1.00 \pm 0.02$ & $1.01 \pm 0.05$ & 0.6849 \\
Relative quadriceps weight (\%) & $1.33 \pm 0.07$ & $1.34 \pm 0.03$ & $1.33 \pm 0.06$ & $1.33 \pm 0.03$ & 0.0896 \\
Relative kidney weight (\%) & $1.80 \pm 0.14$ & $1.83 \pm 0.12$ & $1.83 \pm 0.22$ & $1.83 \pm 0.10$ & 0.7062 \\
Relative heart weight (\%) & $0.52 \pm 0.04$ & $0.52 \pm 0.04$ & $0.50 \pm 0.03$ & $0.51 \pm 0.03$ & 0.4263 \\
Relative lung weight (\%) & $0.60 \pm 0.05$ & $0.62 \pm 0.04$ & $0.60 \pm 0.03$ & $0.61 \pm 0.03$ & 0.8376 \\
Relative EFP weight (\%) & $0.97 \pm 0.366^{\mathrm{b}}$ & $0.74 \pm 0.21 \mathrm{a}$ & $0.73 \pm 0.18^{\mathrm{a}}$ & $0.74 \pm 0.12^{\mathrm{a}}$ & 0.1584 \\
Relative BAT weight (\%) & $0.22 \pm 0.03$ & $0.20 \pm 0.01$ & $0.22 \pm 0.05$ & $0.23 \pm 0.02$ & 0.5144 \\
Relative cecum weight (\%) & $2.02 \pm 0.42$ & $2.01 \pm 0.30$ & $2.00 \pm 0.12$ & $2.03 \pm 0.28$ & 0.9822
\end{tabular}

Data are expressed as mean \pm SD for $n=10$ mice in each group. Data in the same row with different letters ( $a, b)$ differ significantly at $p<0.05$ by one-way ANOVA; EFP: epididymal fat pad; BAT: brown adipose tissue.

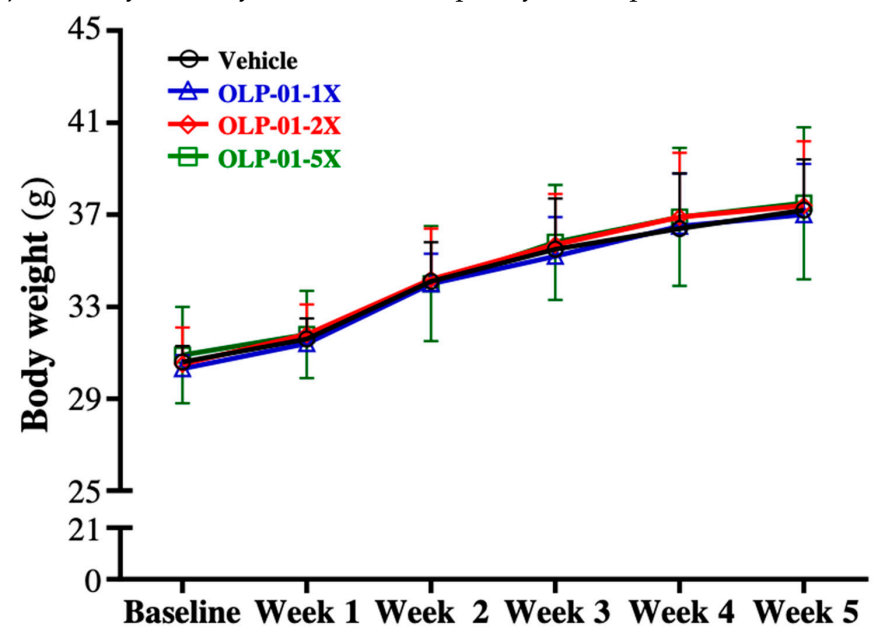

Figure 1. The effect of OLP-01 supplementation on growth curves. Data are expressed as mean \pm SD for $n=10$ mice per group. 


\subsection{Effect of OLP-01 Supplementation on Grip Strength}

As shown in Figure 2A, the mean forelimb grip strengths in the vehicle, OLP-01-1X, OLP-01-2X, and OLP-01-5X groups were $110 \pm 16,133 \pm 8,144 \pm 11$, and $152 \pm 13 \mathrm{~g}$, respectively. This represented a 1.21-fold $(p=0.0002), 1.31$-fold $(p<0.0001)$, and 1.38-fold $(p<0.0001)$ increase in grip strength, respectively, compared with the vehicle group. Relative grip strength (\%) as calculated by normalizing to individual body weight, was also significantly higher with OLP-01 treatment (Figure 2B). Both absolute and relative grip strength measurements showed significant OLP-01 dose-dependent effects $(p<0.0001)$ in trend analysis. Thus, OLP-01 supplementation for 4 weeks was able to improve forelimb grip strength.

(A)

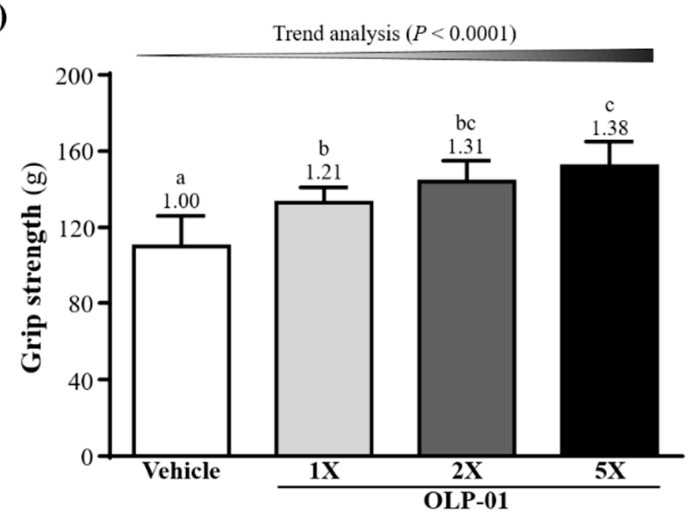

(B)

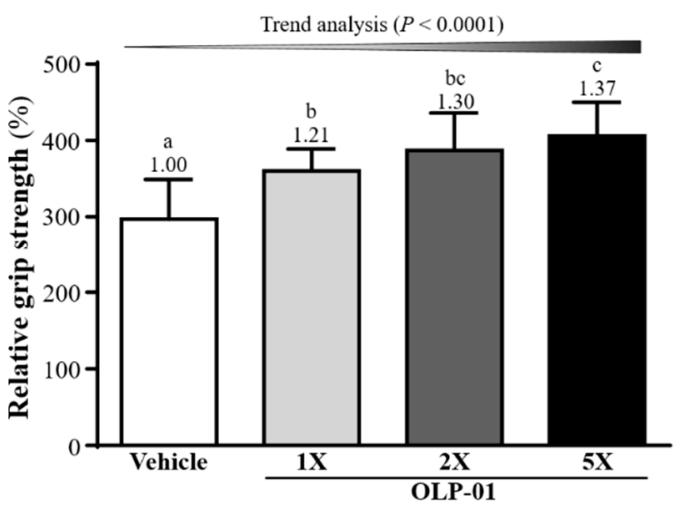

Figure 2. Effect of 4 week OLP-01 supplementation on (A) absolute forelimb grip strength and (B) forelimb grip strength (\%) relative to body weight. Data are expressed as mean \pm SD for $n=10$ mice per group. Different superscript letters $(a, b, c)$ indicate significant difference at $p<0.05$.

\subsection{Effect of OLP-01 Supplementation on Endurance Capacity in the Swim-to-ExhaustionTest}

In Figure 3, the exhaustive swim times in the vehicle, OLP-01-1X, OLP-01-2X, and OLP-01-5X groups were $4.74 \pm 1.33,8.36 \pm 1.42,11.20 \pm 3.65$, and $15.98 \pm 6.20 \mathrm{~min}$, respectively. This corresponded to a 1.77-fold $(p=0.0360), 2.37$-fold $(p=0.0004)$, and 3.37-fold $(p<0.0001)$ increase, respectively, in the OLP-01-1X, OLP-01-2X, and OLP-01-5X groups, compared with the vehicle group. In the trend analysis, maximal swim time was increased dose-dependently with OLP-01 supplementation $(p<0.0001)$.

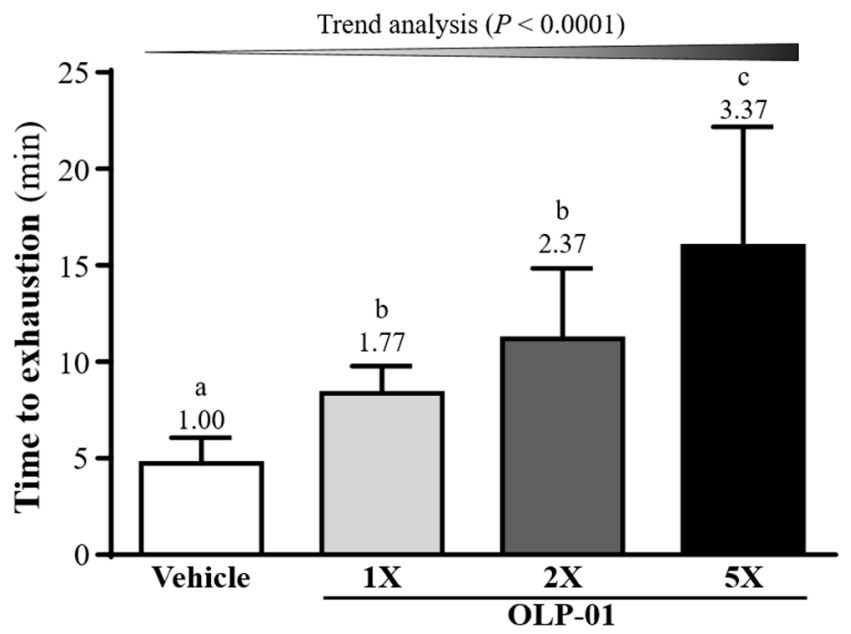

Figure 3. Effect of 4 weeks of OLP-01 supplementation on exhaustive swim times. Data are expressed as mean $\pm \mathrm{SD}$ for $n=10$ mice per group. Different superscript letters $(\mathrm{a}, \mathrm{b}, \mathrm{c})$ indicate significant difference at $p<0.05$. 


\subsection{Effect of OLP-01 Supplementation on Lactate after the 10 Min Swim Test}

After 4 weeks of supplementation, mice underwent a $10 \mathrm{~min}$ swim test to evaluate the levels of lactate, a metabolite highly associated with exercise physiological status. Lactate levels were assessed at three time points: pre-exercise, post- exercise, and after 20 min rest (Table 2). Before swimming and at baseline, there was no significant difference in the levels of blood lactate among the groups. After the 10 min swim test, the levels of blood lactate in vehicle, OLP-01-1X, OLP-01-2X, and OLP-01-5X groups were $6.74 \pm 0.28,5.64 \pm 0.57,5.40 \pm 0.83$, and $5.12 \pm 0.59 \mathrm{mmol} / \mathrm{L}$, respectively. This represented an increase of $16.38 \%(p=0.0002), 19.82 \%(p<0.0001)$, and $24.02 \%(p<0.0001)$ in the OLP-01-1X, OLP-01-2X, and OLP-01-5X groups respectively, compared to the vehicle group. In addition, there was a significant dose-dependent effect on the post-exercise lactate levels $(p<0.0001)$.

After 20 min rest following the swimming test, the blood lactate levels in vehicle, OLP-01-1X, OLP-01-2X, and OLP-01-5X groups were $6.05 \pm 0.25,5.04 \pm 0.654 .76 \pm 0.67$, and $4.53 \pm 0.57 \mathrm{mmol} / \mathrm{L}$, respectively. Compared with the vehicle group, OLP-01-1X, OLP-01-2X, and OLP-01-5X were significantly lower than vehicle by $16.79 \%(p=0.0003), 21.30 \%(p<0.0001)$, and $25.14 \%(p<0.0001)$. This decrease in blood lactate level after rest was shown in the trend analysis to be dose-dependent $(p<0.0001)$. Lactate production rates were significantly changed in all the OLP-01 supplementation groups $(p<0.0001)$ compared to the vehicle group, but lactate clearance rates were not significantly different among the groups.

Table 2. The effect of OLP-01 on lactate levels during acute exercise challenge.

\begin{tabular}{cccccc}
\hline \multirow{2}{*}{ Time Point } & Vehicle & OLP1-01-1X & OLP-01-2X & OLP-01-5X & Trend Analysis \\
\cline { 2 - 5 } & \multicolumn{5}{c}{ Lactate (mmol/L) } \\
\hline Before swimming (A) & $3.42 \pm 0.37$ & $3.43 \pm 0.36$ & $3.41 \pm 0.30$ & $3.43 \pm 0.24$ & 0.7111 \\
After swimming (B) & $6.74 \pm 0.28^{\mathrm{b}}$ & $5.64 \pm 0.57^{\mathrm{a}}$ & $5.40 \pm 0.83^{\mathrm{a}}$ & $5.12 \pm 0.59^{\mathrm{a}}$ & $<0.0001$ \\
After a 20 min rest(C) & $6.05 \pm 0.25^{\mathrm{b}}$ & $5.04 \pm 0.65^{\mathrm{a}}$ & $4.76 \pm 0.67^{\mathrm{a}}$ & $4.53 \pm 0.57^{\mathrm{a}}$ & $<0.0001$ \\
\hline \multicolumn{5}{c}{ Rate of lactate production and clearance } \\
Production rate = B/A & $1.99 \pm 0.23^{\mathrm{c}}$ & $1.64 \pm 0.05^{\mathrm{b}}$ & $1.58 \pm 0.12^{\mathrm{ab}}$ & $1.49 \pm 0.10^{\mathrm{a}}$ & $<0.0001$ \\
Clearance rate $=(\mathrm{B}-\mathrm{C}) / \mathrm{B}$ & $0.10 \pm 0.02$ & $0.11 \pm 0.06$ & $0.12 \pm 0.03$ & $0.12 \pm 0.02$ & 0.0222 \\
\hline
\end{tabular}

The metabolite lactate was assessed for the four groups: vehicle, OLP-01-1X, OLP-01-2X, and OLP-01-5X at three time points. Lactate production rate $(\mathrm{B} / \mathrm{A})$ was calculated as the lactate level after exercise $(\mathrm{B})$ divided by the lactate level before exercise (A). Clearance rate at rest $(\mathrm{B}-\mathrm{C}) / \mathrm{B}$ was defined as the difference in lactate levels immediately after exercise (B) and after rest (C), divided by lactate level after rest (B). Data are expressed as mean \pm SD for $n=10$ mice per group. Values in the same row with different superscript letters $(a, b, c)$ differ significantly, $p<0.05$.

\subsection{Effect of OLP-01 Supplementation on Ammonia and Glucose after the 10 Min Swim Test}

The serum ammonia levels in the mice were measured (Figure 4A) level were found to be $6.05 \pm 0.25$, $5.04 \pm 0.654 .76 \pm 0.67$, and $4.53 \pm 0.57 \mu \mathrm{mol} / \mathrm{L}$ in the vehicle, OLP-01-1X, OLP-01-2X, and OLP-01-5X groups, respectively. The ammonia levels were $20.11 \%(p=0.0044)$ and $28.18 \%(p=0.0001)$ lower in the OLP-01-2X and OLP-01-5X groups compared to the vehicle group. A significant dose-dependent effect on blood ammonia levels was observed $(p<0.0001)$ after the $10 \mathrm{~min}$ swim test. Blood glucose levels were also obtained immediately after the $10 \mathrm{~min}$ swim test (Figure 4B). Only in the OLP-01-5X group was the blood glucose level significantly increased, 1.17-fold $(p=0.0411)$ compared with the vehicle group. However, trend analysis showed a significant dose-dependent effect $(p=0.0078)$ of OLP-01 supplementation on blood glucose levels. 
(A)

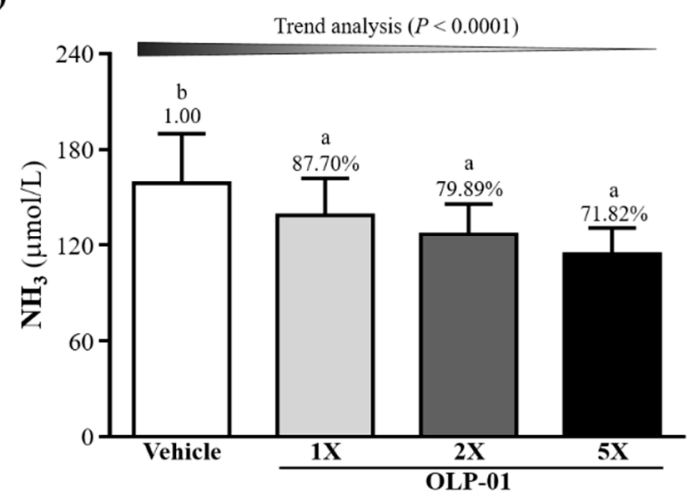

(B)

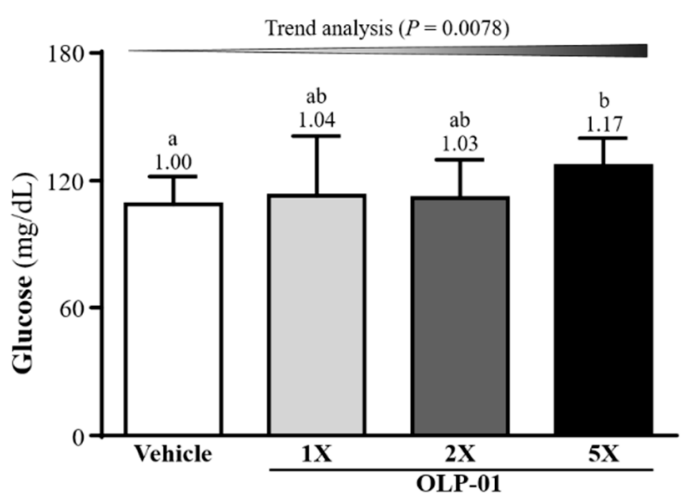

Figure 4. Effect of 4 week OLP-01 supplementation on serum (A) ammonia $\left(\mathrm{NH}_{3}\right)$ and $(\mathbf{B})$ glucose levels after $10 \mathrm{~min}$ acute exercise challenge. Data are expressed as mean $\pm \mathrm{SD}$ for $n=10$ mice per group and the different superscript letters $(\mathrm{a}, \mathrm{b})$ indicate significant difference at $p<0.05$.

\subsection{Effect of OLP-01 Supplementation on BUN and CK after a 90 Min Swim Test and 60 Min Rest Period}

The mouse groups were subjected to an extended 90 min swim test followed by 60 min rest and measured for BUN levels (Figure 5A). OLP-01 supplementation (OLP-01-1X, OLP-01-2X, and OLP-01-5X) significantly decreased the exercise-induced BUN elevation by $11.77 \%(p=0.0054), 12.81 \%$ $(p=0.0027)$, and $16.13 \%(p=0.0003)$, respectively, showing a dose-dependent trend $(p<0.0001)$. The other important injury index, CK, also showed significant difference among the groups (Figure $5 \mathrm{~B}$ ). OLP-01-2X, and OLP-01-5X supplementation could significantly decrease the CK levels by $21.81 \%$ $(p=0.0264)$ and $32.01 \%(p=0.0017)$ compared to the vehicle group, and showed a dose-dependent trend $(p=0.0004)$.

(A)

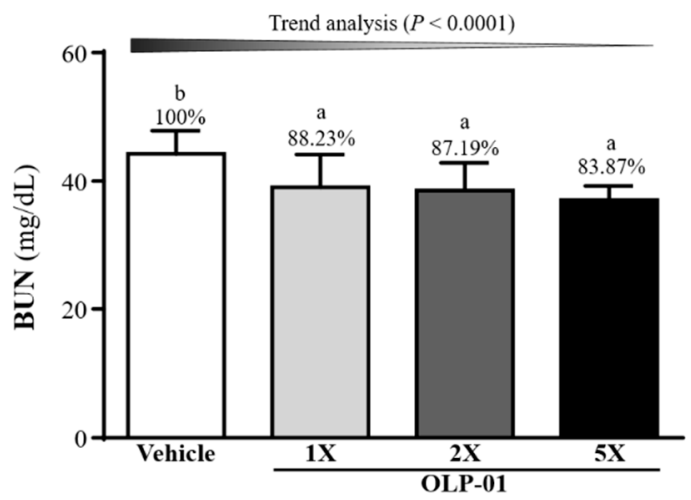

(B)

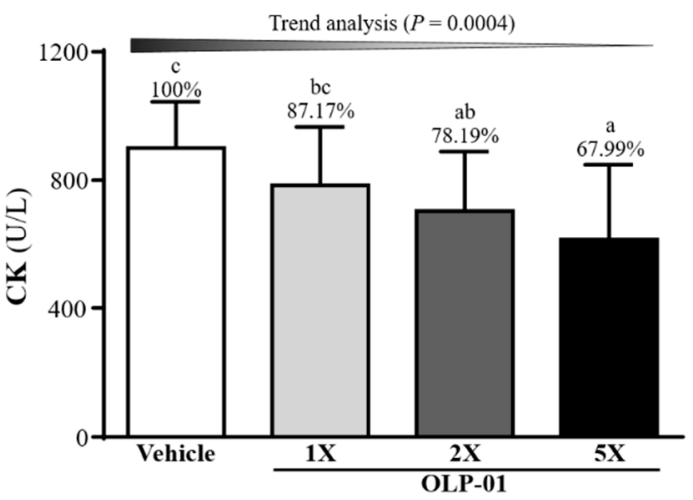

Figure 5. Effect of 4 week OLP-01 supplementation on serum (A) BUN and (B) CK levels after extended exercise challenge. The indicated four groups underwent $90 \mathrm{~min}$ swim exercise and blood was sampled after $60 \mathrm{~min}$ of rest. Data are expressed as mean $\pm \mathrm{SD}$ for $n=10$ mice per group and the different superscript letters $(\mathrm{a}, \mathrm{b}, \mathrm{c})$ indicate significant difference at $p<0.05$.

\subsection{Effect of OLP-01 Supplementation on Liver and Muscle Glycogen}

Glycogen stores in liver were elevated 1.73- $(p=0.0023)$ and 2.38-fold $(p<0.0001)$, respectively, in the OLP-01-2X and OLP-01-5X groups compared with vehicle group (Figure 6A). OLP-01 supplementation (OLP-01-1X, OLP-01-2X, and OLP-01-5X) significantly increased the muscle glycogen levels over the vehicle group 1.71-fold ( $p=0.0004), 2.05$-fold $(p<0.0001)$, and 2.05-fold $(p<0.0001)$, respectively (Figure 6B). A significant dose-dependent effect of OLP-01 on hepatic and muscular glycogen content was observed $(p<0.0001)$. 
(A)

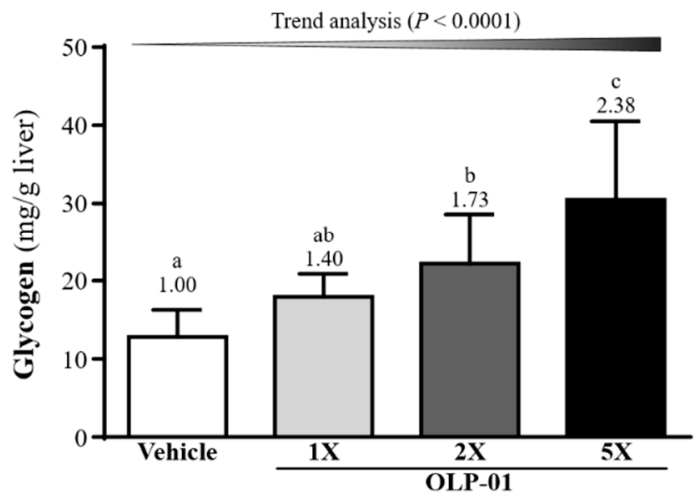

(B)

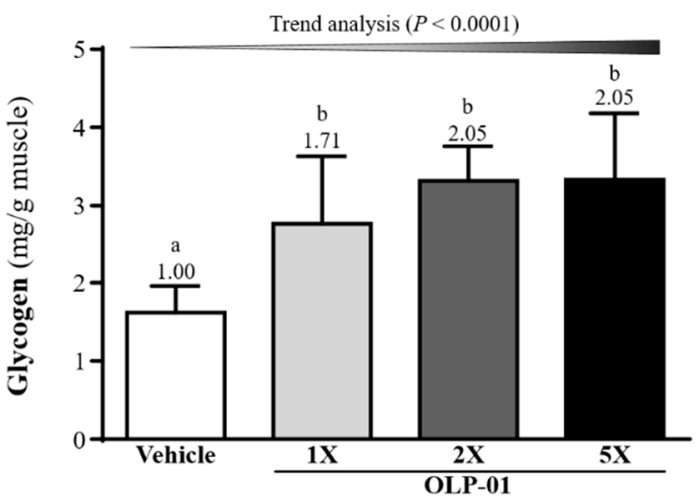

Figure 6. Effect of 4 week OLP-01 supplementation on (A) hepatic glycogen and (B) muscle glycogen levels. Data are expressed as mean $\pm \mathrm{SD}$ for $n=10$ mice per group. Values with different superscript letters $(\mathrm{a}, \mathrm{b}, \mathrm{c})$ are significantly different at $p<0.05$.

\subsection{Effect of OLP-01 Supplementation on Biochemical Profiles at the End of the Study}

We investigated whether the beneficial effects of OLP-01 supplementation on grip strength, exhaustive exercise challenge, and anti-fatigue performance led to changes in related biochemical parameters (Table 3). The liver damage markers AST and ALT were not significantly different between the groups $(p>0.05)$. Mean levels of other biochemical indices, including albumin, TC, TG, CK, BUN, creatinine, UA, TP, and glucose, were also found to be unchanged between the groups $(p>0.05)$. Our results suggest that the doses of OLP-01 supplementation used in the present study are safe, with little effect on biochemical indices.

Table 3. The effects of OLP-01 on biochemical indices at the end of the study.

\begin{tabular}{cccccc}
\hline Parameter & Vehicle & OLP-01-1X & OLP-01-2X & OLP-01-5X & Trend Analysis \\
\hline AST (U/L) & $68 \pm 11$ & $68 \pm 10$ & $69 \pm 27$ & $68 \pm 13$ & 0.9451 \\
ALT (U/L) & $31 \pm 8$ & $32 \pm 10$ & $31 \pm 15$ & $32 \pm 8$ & 0.4689 \\
CK (U/L) & $162 \pm 52$ & $129 \pm 65$ & $120 \pm 69$ & $128 \pm 94$ & 0.0751 \\
GLU (mg/dL) & $229 \pm 21$ & $231 \pm 48$ & $227 \pm 24$ & $228 \pm 26$ & 0.8925 \\
CREA (mg/dL) & $0.38 \pm 0.02$ & $0.37 \pm 0.02$ & $0.37 \pm 0.03$ & $0.38 \pm 0.02$ & 0.9674 \\
BUN (mg/dL) & $23.4 \pm 1.6$ & $23.4 \pm 1.7$ & $23.7 \pm 2.8$ & $22.3 \pm 1.4$ & 0.8665 \\
UA (mg/dL) & $2.6 \pm 0.6$ & $2.5 \pm 0.8$ & $2.5 \pm 0.5$ & $2.5 \pm 0.3$ & 0.8331 \\
TC (mg/dL) & $138 \pm 32$ & $133 \pm 24$ & $135 \pm 15$ & $135 \pm 13$ & 0.9813 \\
TG (mg/dL) & $141 \pm 27$ & $141 \pm 13$ & $141 \pm 20$ & $142 \pm 13$ & 0.5683 \\
ALB (g/dL) & $3.0 \pm 0.1$ & $3.0 \pm 0.2$ & $3.0 \pm 0.2$ & $3.1 \pm 0.1$ & 0.0915 \\
TP (g/dL) & $5.3 \pm 0.2$ & $5.4 \pm 0.2$ & $5.4 \pm 0.3$ & $5.4 \pm 0.2$ & 0.0630 \\
\hline
\end{tabular}

Data are expressed as mean \pm SD for $n=10$ mice in each group. AST, aspartate aminotransferase; ALT, alanine transaminase; $\mathrm{CK}$, creatine kinase; GLU, glucose; CREA, creatinine; $\mathrm{BUN}$, blood urea nitrogen; UA, uric acid; TC, total cholesterol; TG, triacylglycerol; ALB, albumin; TP, total protein.

\subsection{Effect of OLP-01 Supplementation on the Gut Microbiota}

We analyzed the gut microbiota composition using the 16S rRNA genes in the vehicle or OLP-01-treated mice, and observed dramatic changes in the microbial ecology when treated with OLP-01 at the end of experiment. To know whether the supplementation with OLP-01 had altered the gut microbiota, we chose the highest dose of OLP-01-5X to compare with the vehicle. As shown in Table 4 , the genus counts of the gut microbiota of the mice on OLP-01-5X showed slightly increased Lactobacillus (205.66\%), Clostridium (104.51\%), Bifidobacterium (164.25\%), and Lactococcus (571.76\%), and simultaneously decreased Bacillus (92.34\%), Enterococcus (73.95\%), and Akkermansia (92.34\%) compared to vehicle. 
Since OLP-01 is a subspecies of B. longum, it was necessary to count the species of Bifidobacterium. The OLP-01-5X counts in any species of Bifidobacterium were higher than the vehicle (109-1202\%); among then, the B. longum was slightly increased over vehicle by $1210 \%$ ( $p=0.0693$ ).

Table 4. The effects of OLP-01 on the gut microbiota at the end of the study.

\begin{tabular}{ccccc}
\hline Counts & Vehicle & OLP-01-5X & $p$ Value & Compared with Vehicle (\%) \\
\hline & Genus & \\
\hline Lactobacillus & $842.36 \pm 116.85$ & $1732.35 \pm 1290.13$ & 0.2185 & 205.66 \\
Clostridium & $1028.05 \pm 349.22$ & $1074.37 \pm 190.07$ & 0.8235 & 104.51 \\
Bacillus & $581.67 \pm 174.5$ & $537.12 \pm 94.68$ & 0.6693 & 92.34 \\
Enterococcus & $179.41 \pm 105.22$ & $132.67 \pm 59.86$ & 0.4693 & 73.95 \\
Bifidobacterium & $55.05 \pm 7.85$ & $90.42 \pm 30.04$ & 0.0630 & 164.25 \\
Streptococcus & $55.5 \pm 10.55$ & $55.25 \pm 15.36$ & 0.9796 & 99.55 \\
Akkermansia & $68.07 \pm 47.44$ & $27.07 \pm 9.71$ & 0.1413 & 39.77 \\
Lactococcus & $0.23 \pm 0.45$ & $1.3 \pm 1.69$ & 0.2662 & 571.76 \\
\hline & & Species & & \\
\hline Bifidobacterium bombi & $13.85 \pm 4.68$ & $21.22 \pm 10.27$ & 0.2392 & 153.23 \\
Bifidobacterium longum & $1.25 \pm 1.72$ & $15.11 \pm 12.43$ & 0.0693 & 122.05 \\
Bifidobacterium asteroides & $3.15 \pm 1.70$ & $3.84 \pm 1.50$ & 0.5626 & 108.06 \\
Bifidobacterium indicum & $2.51 \pm 1.36$ & $2.71 \pm 1.18$ & 0.8295 & - \\
Bifidobacterium catenulatum & $0 \pm 0$ & $6.22 \pm 2.75$ & $0.0040 *$ & 298.82 \\
Bifidobacterium scardovii & $0.63 \pm 0.56$ & $1.89 \pm 2.22$ & 0.3128 & 196.58 \\
Bifidobacterium stercoris & $0.84 \pm 1.69$ & $1.66 \pm 1.87$ & 0.5412 & 140.76 \\
Bifidobacterium choerinum & $0.77 \pm 0.87$ & $1.08 \pm 0.55$ & 0.5653 & 1092.70 \\
Bifidobacterium subtile & $0.16 \pm 0.33$ & $1.8 \pm 2.19$ & 0.1902 & 563.98 \\
Bifidobacterium adolescentis & $0.17 \pm 0.34$ & $0.95 \pm 1.36$ & 0.3060 & 288.94 \\
Bifidobacterium kashiwanohense & $0.13 \pm 0.26$ & $0.38 \pm 0.46$ & 0.3859 & - \\
Bifidobacterium bifidum & $0 \pm 0$ & $1.26 \pm 2.53$ & 0.3559 & 24.93 \\
Bifidobacterium cuniculi & $0.84 \pm 1.69$ & $0.21 \pm 0.42$ & 0.4938 & - \\
Bifidobacterium merycicum & $0 \pm 0$ & $0.12 \pm 0.23$ & 0.3559 & 0.00 \\
Bifidobacterium magnum & $0.13 \pm 0.27$ & $0 \pm 0$ & 0.3559 & \\
\hline Data are expressed as mean & SD & \\
\hline
\end{tabular}

Data are expressed as mean $\pm \mathrm{SD}$ for $n=10$ mice in each group. Values in the same row with different superscript letters $\left(^{*}\right)$ differ significantly, $p<0.05$, by t test.

\subsection{Effect of OLP-01 Supplementation on Tissue Histology at the End of the Study}

A histological examination of the liver, muscle, heart, kidney, lung, EFP, and BAT obtained from the mice in the different groups was performed at the end of the study. Figure 7 shows representative photomicrographs of organs from the vehicle and OLP-01-supplemented mouse groups. Hematoxylin and eosin (HE) stains of all the liver tissues revealed normal hepatic architecture of hepatocytes, bile duct, and sinusoid. Hypertrophy and hyperplasia were not observed in any of the heart cardiomyocytes or skeletal muscles. Renal sections also revealed normal renal architecture, with a normal appearance of glomeruli, tubules, and interstitial tissues. In addition, there was no difference in the morphology of adipose tissue or fat cell size between the groups. Taken together, the results suggest that OLP-01 supplementation at these doses was safe and did not lead to any adverse effects on major organs or tissues. We analyzed gut microbiota compositions using the 16S rRNA genes in the vehicle or OLP-01-supplementation-treated mice, and observed dramatic changes in the microbial ecology at the end of experiment when treated with OLP-01. To know whether the supplementation of OLP-01 bacteria had altered the intestinal flora, we chose the highest dose of OLP-01-5X to compare with the vector. 


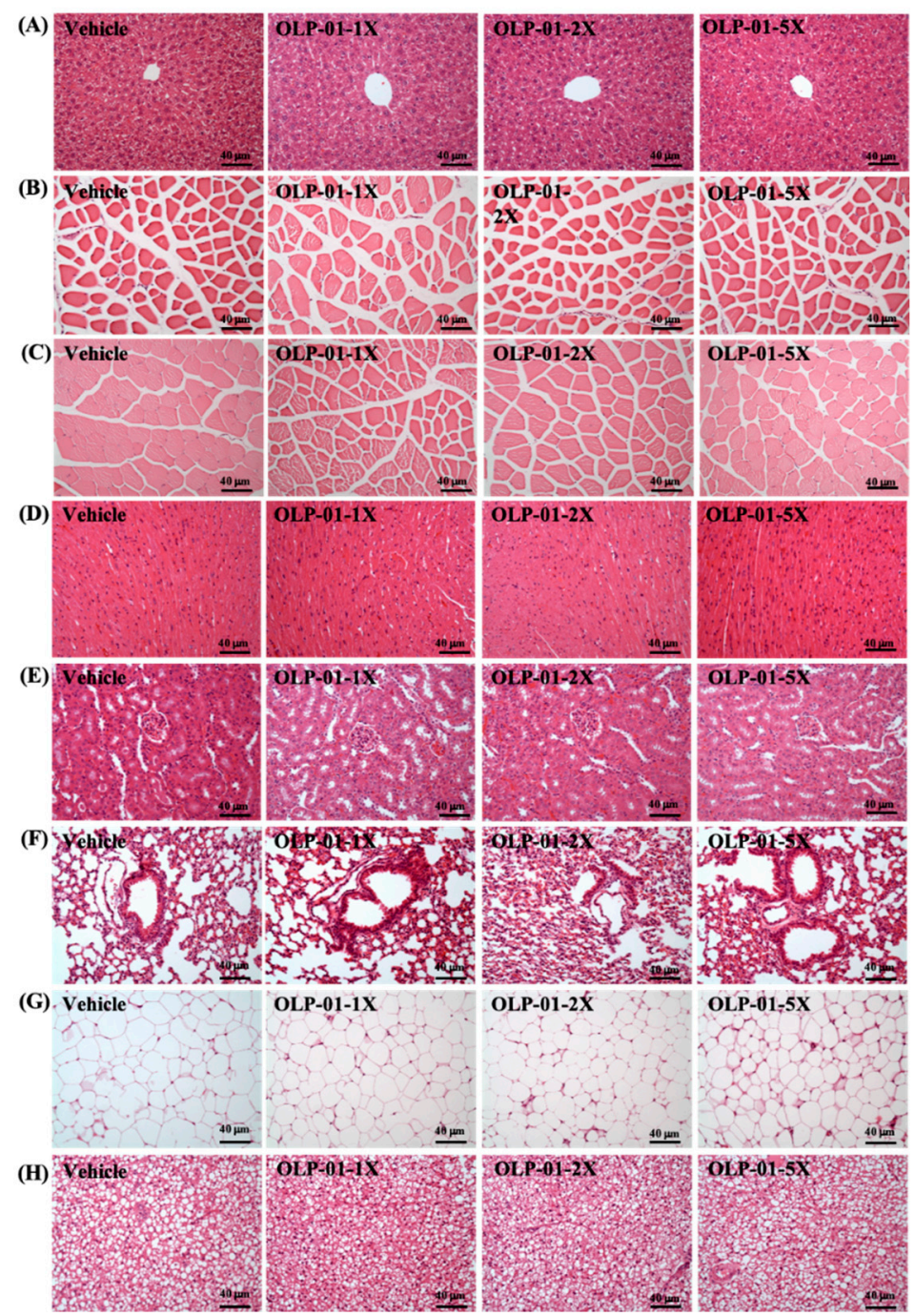

Figure 7. Effect of OLP-01 supplementation on histomorphology of the (A) liver, (B) muscle, (C) quadricep muscles, (D) heart, (E) kidney, (F) lung, (G) adipocyte tissue, and (H) BAT tissue in mice. Specimens were photographed under a light microscope (H\&E stain, magnification: 200×; bar, $40 \mu \mathrm{m})$.

\section{Discussion}

In this study, we used B. longum (OLP-01) as a supplement to study its physiological effects on exercise performance and related parameters. We have shown that OLP-01 supplementation significantly promotes grip strength and exercise endurance performance without any training intervention. Biochemical indicators of fatigue and injury after exercise challenge, such as blood levels of lactate, $\mathrm{BUN}, \mathrm{NH}_{3}$, and $\mathrm{CK}$ were found to be significantly reduced, while the recovery from exercise fatigue was accelerated with OLP-01 supplementation. This suggests a benefit of OLP-01 in improving physiological adaptation.

The effect of probiotics on exercise performance may vary according to the probiotic studied; different strains have different mechanisms of action, which have not been confirmed. Previous research has pointed out that prebiotic or probiotic supplementation could increase intestinal SCFA content $[27,28]$, such as acetate, $n$-butyrate, and propionate. This could regulate host energy balance and increase nutrient availability [29]. In the case of Bifidobacteria, these monosaccharides are converted to intermediates of the hexose fermentation pathway, also known as the fructose-6-phosphate shunt or 'bifid' shunt $[30,31]$. They are ultimately converted to SCFAs and other organic compounds, some of which may be beneficial to the host. SCFAs are absorbed by intestinal mucosa, relatively high in caloric content, and metabolized by the colonocytes and hepatocytes. In colonocytes, butyrate is 
transported to mitochondria, where it undergoes fatty-acid oxidation (FAO) in aerobic conditions and becomes acetyl-CoA. The acetyl-CoA enters the Krebs cycle to produce NADH, which enters the electron transport chain and leads to adenosine triphosphate (ATP) and $\mathrm{CO}_{2}$ production [32]. Interestingly, n-butyrate could play an important role in peroxisome proliferator-activated receptor gamma coactivator- $1 \alpha$ (PGC- $1 \alpha$ ) activation; it has been shown to dramatically increase in skeletal muscles in response to exercise [33], and induction leads to a transformation of skeletal muscle fibers from type II (glycolytic) to type I (oxidative). These type I fibers are rich in mitochondria and stimulate FAO for ATP production [34]. Therefore, it is possible that PGC-1 $\alpha$ gene expression may be considered a potential biomarker of mitochondrial function during endurance training.

Forelimb grip strength is a physical test that reflects the overall health of the musculoskeletal system and can be used to evaluate motor-associated coordination and adaptation in neurological studies [35]. In this study, although the muscle mass of the OLP-01 and vehicle groups did not differ with 4 weeks of supplementation (Table 1), the forelimb grip strength was shown to increase significantly in the OLP-01 groups (Figure 2). In general, programmed exercise training is required to improve endurance capacity and grip strength [36]. Although no training program was implemented in this study, our experimental results indicate that endurance capacity may be improved and exercise-induced physical fatigue ameliorated by 4 weeks of OLP-01 supplementation (Figure 3).

Exercise related indexes like lactate, BUN, CK, and glucose are widely used to assess exercise physiological status. The energy metabolism pathway contains the phosphagen system, glycolysis, and the aerobic system, which are dependent on exercise intensity and duration for initiation [25]. Blood lactate is the glycolysis product of carbohydrate under anaerobic conditions, when glycolysis is the main energy source [37]. Hydrogen ions are produced together with lactate, probably due to the dissociation of lactic acid. A decrease in $\mathrm{pH}$ in blood and muscle tissue leads to inhibition of muscle contraction and glycolysis, as well as various deleterious biochemical, metabolic, and physiological side effects [38]. However, previous studies have pointed out that probiotics can improve the production of lactic acid during exercise and convert it into SCFAs, especially propionic acid and butyrate, and provide the energy needed for muscles during exercise [39]. We have shown that OLP-01 supplementation could significantly decrease exercise-induced lactate levels and improve lactate production rate (Table 2). Therefore, we suggest that the OLP-01 supplement is beneficial for the removal and utilization of lactic acid, thereby reducing the development of fatigue.

Another important indicator of fatigue is blood ammonia. During exercise, ammonia is produced and accumulates in skeletal muscle when adenosine monophosphate (AMP) is deaminated to inosine monophosphate (IMP) by AMP deaminase (AMPD) during resynthesis of ATP [26]. The ingestion of Bifidobacterium together with lactulose has been shown to assist in reestablishing the balance of the gut flora. This was accompanied by a decrease in fecal $\mathrm{pH}$ and reduction of ammonia and free phenols in the blood [40]. As seen in Figure 4A, supplementing mice with 4 weeks of OLP-01 effectively reduced blood ammonia concentration after exercise. Ammonia may be metabolized as BUN via the urea cycle [41], thus BUN could be considered a biomarker for ATP metabolism, and not just a marker for kidney function. After the $90 \mathrm{~min}$ swim test and $60 \mathrm{~min}$ rest period, BUN was shown to be significantly decreased in the OLP-01-supplemented mice (Figure 5A).

Glucose is stored as glycogen, which is an important energy source for ATP production and is mainly present in the liver and muscle tissues. Glycogen is able to complement the consumption of blood glucose during exercise and helps to maintain blood glucose levels within the physiological range [42]. There is a high correlation between glycogen storage and carbohydrate uptake and metabolism [43]. Previous studies have shown that $B$. longum is able to transport a variety of disaccharides and oligosaccharides, e.g., oligofructose, which are described as growth-promoting prebiotics. More than half of the transport systems are ATP-dependent ATP-binding cassette (ABC)-type permeases. B. longum has a glucose-specific phosphoenolpyruvate-dependent sugar phosphotransferase system (PTS), while two other Bifidobacterium species (lactis and bifidum) have a fructose-6-phosphate-forming fructose-PTS instead. It has become obvious that apart from a few exceptions, most carbohydrate 
systems are closely related to those from other actinomycetes [44]. In this study, supplementation with OLP-01 effectively increased liver and muscle glycogen storage (Figure 6A,B), improving glycogen degradation and exercise performance.

Probiotics may be used as a supplement to meet most of our basic nutritional and health requirements. Previous studies have shown B. longum to be a safe bacteria strain [11]. We also observed the safety of B. longum in 4 weeks of OLP-01 supplementation in the mice. Serum metabolic markers, including liver and renal function tests, were found to be within the normal range, even after several exercise challenges. In pathological sections, we also did not detect any gross abnormalities or obvious lesions in the various tissues and organs. Taken together, we believe that the supplementation of OLP-01 (B. longum) improve gastrointestinal symptoms, accelerate recovery fatigue, and improve exercise performance, and should not lead to any safety concerns.

\section{Conclusions}

In the current study, we found that four weeks' supplementation of OLP-01, a bacterial strain isolated from a top weightlifting athlete, could increase liver glycogen level and improve both grip strength and endurance in mice. In addition, OLP-01 showed anti-fatigue properties by lowering serum lactate, ammonia, and CK levels in a dosage-dependent manner. We suggest that OLP-01 could be used as a supplement to modify gut microbiota and enhance exercise performance while mitigating fatigue. The mechanism and pathway activation by OLP-01 warrants further investigation.

Author Contributions: H.-L.C., P.-S.H. and C.-C.H. designed the experiments. M.-C.L., H.-H.H. and Y.-J.H. carried out the laboratory experiments. W.-L.C., H.-L.C., P.-S.H., Y.-S.C. and C.-C.H. contributed reagents, material, and analysis platforms. M.-C.L., H.-H.H., Y.-J.H., and C.-C.H. analyzed the data. M.-C.L., Y.-S.C. and C.-C.H. interpreted the results, prepared figures, wrote and revised the manuscript.

Funding: This research received no external funding.

Acknowledgments: The authors are grateful to Chin-Shan Ho for technical assistance in measuring the forelimb grip strength. We thank Chien-Chao Chiu for technical assistance in the histological examinations.

Conflicts of Interest: The authors declare no conflict of interest.

\section{References}

1. Schmidt, T.S.B.; Raes, J.; Bork, P. The human gut microbiome: From association to modulation. Cell 2018, 172, 1198-1215. [CrossRef] [PubMed]

2. Barton, W.; Penney, N.C.; Cronin, O.; Garcia-Perez, I.; Molloy, M.G.; Holmes, E.; Shanahan, F.; Cotter, P.D.; O'Sullivan, O. The microbiome of professional athletes differs from that of more sedentary subjects in composition and particularly at the functional metabolic level. Gut 2018, 67, 625-633. [CrossRef] [PubMed]

3. Clarke, S.F.; Murphy, E.F.; O’Sullivan, O.; Lucey, A.J.; Humphreys, M.; Hogan, A.; Hayes, P.; O’Reilly, M.; Jeffery, I.B.; Wood-Martin, R.; et al. Exercise and associated dietary extremes impact on gut microbial diversity. Gut 2014, 63, 1913-1920. [CrossRef] [PubMed]

4. Havenaar, R.; Huis, J.H. Probiotics: A general view. In The Lactic Acid Bacteria Volume 1; Springer: Boston, MA, USA, 1992; pp. 151-170.

5. Currò, D.; Ianiro, G.; Pecere, S.; Bibbò, S.; Cammarota, G. Probiotics, fibre and herbal medicinal products for functional and inflammatory bowel disorders. Br. J. Pharmacol. 2017, 174, 1426-1449. [CrossRef] [PubMed]

6. Kim, S.; Huang, E.; Park, S.; Holzapfel, W.; Lim, S.D. Physiological Characteristics and Anti-obesity Effect of Lactobacillus plantarum K10. Korean J. Food Sci. Anim. Resour. 2018, 38, 554-569. [PubMed]

7. Grover, S.; Rashmi, H.M.; Srivastava, A.K.; Batish, V.K. Probiotics for human health -new innovations and emerging trends. Gut Pathog. 2012, 4, 15. [CrossRef] [PubMed]

8. Karimi, G.; Sabran, M.R.; Jamaluddin, R.; Parvaneh, K.; Mohtarrudin, N.; Ahmad, Z.; Khazaai, H.; Khodavandi, A. The anti-obesity effects of Lactobacillus casei strain Shirota versus Orlistat on high fat diet-induced obese rats. Food Nutr. Res. 2015, 59, 29273. [CrossRef] 
9. Son, S.H.; Yang, S.J.; Jeon, H.L.; Yu, H.S.; Lee, N.K.; Park, Y.S.; Paik, H.D. Antioxidant and immunostimulatory effect of potential probiotic Lactobacillus paraplantarum SC61 isolated from Korean traditional fermented food, jangajji. Microb. Pathog. 2018, 125, 486-492. [CrossRef]

10. Hill, C.; Guarner, F.; Reid, G.; Gibson, G.R.; Merenstein, D.J.; Pot, B.; Morelli, L.; Canani, R.B.; Flint, H.J.; Salminen, S.; et al. Expert consensus document. The International Scientific Association for Probiotics and Prebiotics consensus statement on the scope and appropriate use of the term probiotic. Nat. Rev. Gastroenterol. Hepatol. 2014, 11, 506-514. [CrossRef]

11. George Kerry, R.; Patra, J.K.; Gouda, S.; Park, Y.; Shin, H.S.; Das, G. Benefaction of probiotics for human health: A review. J. Food Drug Anal. 2018, 26, 927-939. [CrossRef]

12. Vemuri, R.; Shinde, T.; Shastri, M.D.; Perera, A.P.; Tristram, S.; Martoni, C.J.; Gundamaraju, R.; Ahuja, K.D.K.; Ball, M.; Eri, R. A human origin strain Lactobacillus acidophilus DDS-1 exhibits superior in vitro probiotic efficacy in comparison to plant or dairy origin probiotics. Int. J. Med. Sci. 2018, 15, 840-848. [CrossRef] [PubMed]

13. Nagpal, R.; Wang, S.; Ahmadi, S.; Hayes, J.; Gagliano, J.; Subashchandrabose, S.; Kitzman, D.W.; Becton, T.; Read, R.; Yadav, H. Human-origin probiotic cocktail increases short-chain fatty acid production via modulation of mice and human gut microbiome. Sci. Rep. 2018, 8, 12649. [CrossRef] [PubMed]

14. Lee, J.H.; O'Sullivan, D.J. Genomic insights into bifidobacteria. Microbiol. Mol. Biol. Rev. 2010, 74, 378-416. [CrossRef] [PubMed]

15. Garrido, D.; Ruiz-Moyano, S.; Jimenez-Espinoza, R.; Eom, H.J.; Block, D.E.; Mills, D.A. Utilization of galactooligosaccharides by Bifidobacterium longum subsp. infantis Isolates. Food Microbiol. 2013, 33, $262-270$. [CrossRef] [PubMed]

16. Yuan, J.; Zhu, L.; Liu, X.; Li, T.; Zhang, Y.; Ying, T.; Wang, B.; Wang, J.; Dong, H.; Feng, E.; et al. A proteome reference map and proteomic analysis of Bifidobacterium longum NCC2705. Mol. Cell Proteom. 2006, 5, 1105-1118. [CrossRef] [PubMed]

17. Inoue, T.; Kobayashi, Y.; Mori, N.; Sakagawa, M.; Xiao, J.Z.; Moritani, T.; Sakane, N.; Nagai, N. Effect of combined bifidobacteria supplementation and resistance training on cognitive function, body composition and bowel habits of healthy elderly subjects. Benef. Microbes 2018, 10, 1-12. [CrossRef] [PubMed]

18. Ibrahim, N.S.; Muhamad, A.S.; Ooi, F.K.; Meor-Osman, J.; Chen, C.K. The effects of combined probiotic ingestion and circuit training on muscular strength and power and cytokine responses in young males. Appl. Physiol. Nutr. Metab. 2018, 43, 180-186. [CrossRef] [PubMed]

19. Kang, D.; Li, Z.; Ji, G.E. Anti-Obesity Effects of a Mixture of Fermented Ginseng, Bifidobacterium longum BORI, and Lactobacillus paracasei CH88 in High-Fat Diet-Fed Mice. J. Microbiol. Biotechnol. 2018, 28, 688-696. [CrossRef] [PubMed]

20. Jang, S.E.; Jeong, J.J.; Kim, J.K.; Han, M.J.; Kim, D.H. Simultaneous Amelioratation of Colitis and Liver Injury in Mice by Bifidobacterium longum LC67 and Lactobacillus plantarum LC27. Sci. Rep. 2018, 8, 7500. [CrossRef]

21. Chen, Y.M.; Wei, L.; Chiu, Y.S.; Hsu, Y.J.; Tsai, T.Y.; Wang, M.F.; Huang, C.C. Lactobacillus plantarum TWK10 Supplementation Improves Exercise Performance and Increases Muscle Mass in Mice. Nutrients 2016, 8, 205. [CrossRef]

22. Hsu, Y.J.; Huang, W.C.; Lin, J.S.; Chen, Y.M.; Ho, S.T.; Huang, C.C.; Tung, Y.T. Kefir Supplementation Modifies Gut Microbiota Composition, Reduces Physical Fatigue, and Improves Exercise Performance in Mice. Nutrients 2018, 10, 862. [CrossRef]

23. Kan, N.W.; Lee, M.C.; Tung, Y.T.; Chiu, C.C.; Huang, C.C.; Huang, W.C. The Synergistic Effects of Resveratrol combined with Resistant Training on Exercise Performance and Physiological Adaption. Nutrients 2018, 10, 1360. [CrossRef] [PubMed]

24. Wei, L.; Wen, Y.T.; Lee, M.C.; Ho, H.M.; Huang, C.C.; Hsu, Y.J. Effects of isolated soy protein and strength exercise training on exercise performance and biochemical profile in postpartum mice. Metabolism 2019, 94, 18-27. [CrossRef]

25. Huang, W.C.; Huangm, H.Y.; Hsu, Y.J.; Su, W.H.; Shen, S.Y.; Lee, M.C.; Lin, C.L.; Huang, C.C. The Effects of Thiamine Tetrahydrofurfuryl Disulfide on Physiological Adaption and Exercise Performance Improvement. Nutrients 2018, 10, 8. [CrossRef] [PubMed]

26. Nichols, A.W. Probiotics and athletic performance: A systematic review. Curr. Sports Med. Rep. 2007, 6, 269-273. [CrossRef] [PubMed] 
27. LeBlanc, J.G.; Chain, F.; Martín, R.; Bermúdez-Humarán, L.G.; Courau, S.; Langella, P. Beneficial effects on host energy metabolism of short-chain fatty acids and vitamins produced by commensal and probiotic bacteria. Microb. Cell Fact. 2017, 16, 79. [CrossRef]

28. Samuel, B.S.; Shaito, A.; Motoike, T.; Rey, F.E.; Backhed, F.; Manchester, J.K.; Hammer, R.E.; Williams, S.C.; Crowley, J.; Yanagisawa, M.; et al. Effects of the gut microbiota on host adiposity are modulated by the short-chain fatty-acid binding G protein-coupled receptor, Gpr41. Proc. Natl. Acad. Sci. USA 2008, 105, 16767-16772. [CrossRef]

29. de Vries, W.; Stouthamer, A.H. Pathway of glucose fermentation in relation to the taxonomy of bifidobacteria. J. Bacteriol. 1967, 93, 574-576.

30. Sneath, P.H.; Mair, N.S.; Sharpe, M.E.; Holt, J.G. Bergey's Manual of Systematic Bacteriology; Williams \& Wilkins: Philadelphia, PA, USA, 1986; Volume 2.

31. Donohoe, D.R.; Garge, N.; Zhang, X.; Sun, W.; O'Connell, T.M.; Bunger, M.K.; Bultman, S.J. The microbiome and butyrate regulate energy metabolism and autophagy in the mammalian colon. Cell Metab. 2011, 13, 517-526. [CrossRef]

32. Gao, Z.; Yin, J.; Zhang, J.; Ward, R.E.; Martin, R.J.; Lefevre, M.; Cefalu, W.T.; Ye, J. Butyrate improves insulin sensitivity and increases energy expenditure in mice. Diabetes 2009, 58, 1509-1517. [CrossRef]

33. Al-Obaidi, S.; Al-Sayegh, N.; Nadar, M. Smoking impact on grip strength and fatigue resistance: Implications for exercise and hand therapy practice. J. Phys. Act. Health 2014, 11, 1025-1031.

34. Clark, A.; Mach, N. The Crosstalk between the Gut Microbiota and Mitochondria during Exercise. Front. Physiol. 2017, 8, 319. [CrossRef]

35. Nair, A.B.; Jacob, S. A simple practice guide for dose conversion between animals and human. J. Basic Clin. Pharm. 2016, 7, 27-31. [CrossRef]

36. Chen, Y.M.; Lee, H.C.; Chen, M.T.; Huang, C.C.; Chen, W.C. Dehydroepiandrosterone supplementation combined with Weight-Loading Whole-Body Vibration Training (WWBV) affects exercise performance and muscle glycogen storage in middle-aged C57BL/6 mice. Int. J. Med. Sci. 2018, 15, 564-573. [CrossRef]

37. Cairns, S.P. Lactic acid and exercise performance. Sports Med. 2006, 36, 279-291. [CrossRef]

38. Hsu, Y.J.; Huang, W.C.; Chiu, C.C.; Liu, Y.L.; Chiu, W.C.; Chiu, C.H.; Chiu, Y.S.; Huang, C.C. Capsaicin Supplementation Reduces Physical Fatigue and Improves Exercise Performance in Mice. Nutrients 2016, 8, 648. [CrossRef]

39. Scheiman, J.; Luber, J.M.; Chavkin, T.A.; MacDonald, T.; Tung, A.; Pham, L.D.; Wibowo, M.C.; Wurth, R.C.; Punthambaker, S.; Tierney, B.T.; et al. Meta-omics analysis of elite athletes identifies a performance-enhancing microbe that functions via lactate metabolism. Nat. Med. 2019, 25, 1104-1109. [CrossRef]

40. Deguchi, Y.; Makino, K.; Iwabuchi, A.; Watanuki, M.; Yamashita, T. Selection of ammonia-assimilating bifidobacteria and their effect on ammonia levels in rat caecal contents and blood. Microb. Ecol. Health Dis. 1993, 6, 85-94. [CrossRef]

41. Korzeniewski, B. AMP deamination delays muscle acidification during heavy exercise and hypoxia. J. Biol. Chem. 2006, 281, 3057-3066. [CrossRef]

42. Ørtenblad, N.; Westerblad, H.; Nielsen, J. Muscle glycogen stores and fatigue. J. Physiol. 2013, 591, 4405-4413. [CrossRef]

43. Hearris, M.A.; Hammond, K.M.; Fell, J.M.; Morton, J.P. Regulation of Muscle Glycogen Metabolism during Exercise: Implications for Endurance Performance and Training Adaptations. Nutrients 2018, 10, 298. [CrossRef]

44. Parche, S.; Amon, J.; Jankovic, I.; Rezzonico, E.; Beleut, M.; Barutçu, H.; Schendel, I.; Eddy, M.P.; Burkovski, A.; Arigoni, F; et al. Sugar transport systems of Bifidobacterium longum NCC2705. J. Mol. Microbiol. Biotechnol. 2007, 12, 9-19. [CrossRef]

(C) 2019 by the authors. Licensee MDPI, Basel, Switzerland. This article is an open access article distributed under the terms and conditions of the Creative Commons Attribution (CC BY) license (http://creativecommons.org/licenses/by/4.0/). 